\title{
Data Forest: A Collaborative Version
}

Ronan Jamieson, Adrian Haffegee, Priscilla Ramsamy, and Vassil Alexandrov

Advanced Computing and Emerging Technologies Centre, The School of Systems Engineering, University of Reading,

Reading, RG6 6AY, United Kingdom

r.jamieson@reading.ac.uk

\begin{abstract}
As we increase our ability to produce and store ever larger amounts of data, it is becoming increasingly difficult to understand what the data is trying to tell us. Not all the data we are currently producing can easily fit into traditional visualization methods. This paper presents a new and novel visualization technique based on the concept of a Data Forest. Our Data Forest has been developed to be utilised by virtual reality (VR) systems. VR is a natural information medium. This approach can easily be adapted to be used in collaborative environments. A test application has been developed to demonstrate the concepts involved and a collaborative version tested.
\end{abstract}

Keywords: Data Forest, collaborative virtual environments, data visualization.

\section{Introduction}

The search to efficiently extract information and meaning from data has preoccupied mankind. It has lead humans to try many different methods of presenting the data from hieroglyphics to printed tables, but they mainly rely on our primary sense which is sight. This is due to the fact that humans have a highly developed visual system, that has evolved over time from the need to survive (i.e. tracking food sources) to more complex requirements like entertainment (e.g. pattern matching on Rubik's cubes). Humans excel at pattern recognition and are capable of gaining a better insight and understanding from this. Based on this ability, we find it possible to make intuitive decisions even if there is incomplete or missing data. Any method that uses a 3D object approach will be more effective, due to the fact that it is more natural and intuitive considering this is how humans view near objects.

Current methods used to extract knowledge from variable data sets that do not fit into traditional structures do not necessarily scale well to large multidimensional levels. Therefore a new approach is required that overcomes this problem and utilises the recent advances in computer and graphics technology. A VR Data Forest fulfils this requirement. VR is a discipline that enables users to view large complex data sets in an intuitive and natural manner. For example a $3 \mathrm{D}$ object can be a graphical representation of a particular data value. Immersive 
Projection Technology (IPT) systems like CAVEs 3] or powerwalls, are becoming increasingly popular VR systems for high-end visualization and analysis of large multi-parameter data sets. The feeling of immersion, coupled with natural forms of interaction are particularly useful when using data sets.

$\mathrm{VR}$ is currently being utilised by a wide range of industries to gain an insight into their data sets, these industries range from gas and oil (e.g. seismic data) to the medical profession (e.g. pre-surgery planning). Using VR it is possible to network Virtual Environments (VE) together and create a Collaborative Virtual Environment (CVE). This has be been defined as "a software system in which multiple users interact with each other in real time, even though those users may be located around the world" [1].

This ability to collaboratively work with others has expanded the skill base that can become involved in a project, by allowing the location barrier to be removed. Now experts from different locations can efficiently and effectively work on collaborative projects. Therefore it is becoming a more common project requirement. The next section describes the related work in collaborative environments and data visualization, Section 3 gives a detailed explanation of the concepts involved. Section 4 examines some of the potential application area. Then Section 5 outlines the conclusions and discusses any future work.

\section{Related Work}

The most common approach to developing CVE that allow users to interact with other users and data, has been to build applications on top of different networking platforms and protocols. In VR this has been with either COVEN 8], DIVE[1] or CAVERN 6]. COVEN has it roots in DIVE, as it took lessons learned from that effort and other collaborative environments. DIVE uses a peer to peer approach to networking the environments, this platform allows for the creation of interactive environments, but uses other software to enable its rendering. CAVERN has an efficient database/network library, but needs additional applications to create a CVE (e.g. CoAnim[2] which then limits its functionality.

The scientific community have been developing different approaches to analysing and exploring large multi-dimensional data sets for a number of years. These have resulted in highly advanced tool sets for their needs, for example application like VTK[9], AVS[12] and Open DX [7] are available and widely used. Other communities have relied on traditional methods, namely data mining techniques. These rely on statistical techniques, genetic algorithms and neural networks to uncover the relevant data. Then the data is usually visualized using $2 \mathrm{D}$ graphs, spread sheets, or quasi-3D structures but using a 2D medium (e.g. desktop monitor or projector). A further disadvantage to data mining techniques is the lack of interaction and rapid feedback to the user. Examples of data mining applications that take advantage of visualization techniques are MineSet [0] and Iris Explorer 4], but they use 2D \& quasi-3D structures to represent the data. Furthermore these methods do not scale well to the large multi-dimensional data set. New methods are required that utilise the advantages of new technology (e.g. VR). 


\section{A Data Forest}

A Data Forest is a graphical representation of complex data using the concept of data trees (see Figure 1 (a) \& (b)) to represent the data. Each data tree consists of a trunk and crown; these represent different parameters of the data set that has been chosen. Dependant on which type of VR system the user has access to, the user can then walks and/or navigate through the forest. This will allow that user to effectively spot trends, discover relationships or uncover unusual patterns in the trees. This can then lead to further examination of the underlying data. To increase the flexibility of this approach the forest can either be static or dynamic, this will be depend on the nature of the data to be visualized. A dynamic forest will allow for the use of time variant information. A data tree has been chosen due to the fact that it is a simple object that people are familiar with. People can relate to making decision on the size and shape of a tree relative to another tree.

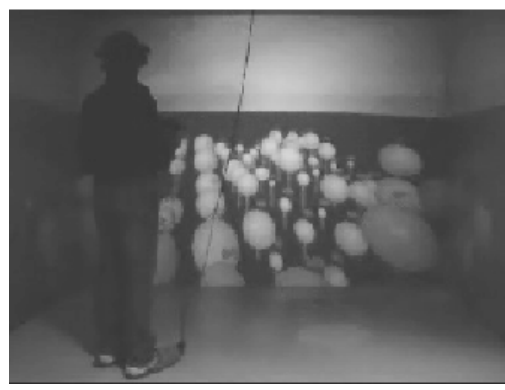

(a)

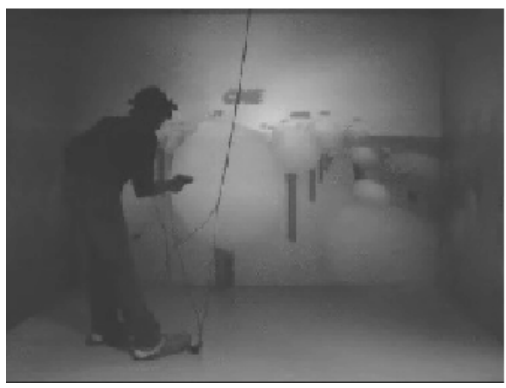

(b)

Fig. 1. Data Forest - (a) Flying over (b) Inside Forest

Up to eight different parameters can be visualized in any one data tree. This number can be achieved by matching the physical properties of the tree (i.e. trunk and crown) to different parameters within the data set,

- position of the tree

- height of trunk

- width of trunk

- colour of trunk

- transparency of the trunk

- radius of crown,

- colour of crown

- transparency of crown

It is possible to expand the number of parameters even further by using all axis of the tree position and inverting the tree (i.e. crown below the trunk). 
Therefore we do not have to have a flat forest; the trees could be positioned at various heights and depths.

Graphically a tree is a simple object, as it just consists of two primitive objects a sphere and cylinder. Simple objects are desirable due to the fact that they retain their shape if a low graphical computation is required (i.e. a tree with a low numbers of polygons will still resemble a tree object). Therefore when required to render large numbers of them, it is still possible to retain a fast and smooth environment. This is an important feature that allows our approach to be used by a wider range of hardware platforms. By using VR we are able to develop an interactive $\mathrm{VE}$, this gives us the ability to interact with the data in a more natural manor, especially if we are using an Immersive VR system. From this interaction it is possible to represent different level/layers of abstraction of the data, by creating different Data Forests. "Drilling down" is a common term in mentioned in data mining techniques, but we have taken the approach of "exposing the roots" of the data tree. Users can choose a particular tree that they are interested by using their input device, then using a 3D menu (see Figure 3(a)) choose to further examine the related data. This gives the users an opportunity to examine in a more detailed fashion the data that is relevant to the chosen tree (see Figure 3(b)). It is possible to return to the original Data Forest or descend to a lower layer (i.e. assuming there are further layers below the current layer) by using the 3D menu.

Figure 2 (see below) outlines a simple overview of the test application that has been developed. The networking of the Data Forest VEs was a logical step, which benefits the users. This has been achieved using the structure proposed in Collaborative Virtual Visualization (CVV) [5], this also allows us to be hardware and platform independent. By using the CVV approach we will represent our remote users as avatars, communicate with them via audio and video links. The avatars will be mapped to the remote users position and orientation, so we will be capable of determining gestures (e.g. pointing to a particular tree) and location within the Data Forest. User decisions in each environment (i.e. remote and local) will be reflected in the connected environment by the use of event messages.

A data filter can be applied to the input data to check the validity of the data; this filter is in the form of a reference table. Once the data has been verified a data structure for each tree is created containing all the data that is relevant to that tree (e.g. trunk size, tree name, etc). These data structure are used in the rendering of the CVE, event messages that allow the environments to be updated to reflect the user's activities will be passed over the network, e.g. when a user chooses a tree to examine further. An event message will be passed to the other remote users application with the all the relevant information to inform the application that a change has occurred. The application will update the remote users VE to reflect this decision. A design aim has been to create a modular application that will easily allow specific functionality can be added, depending on the data type to be analysed. An example of this is discussed in the application section concerning Stock Market Data. 


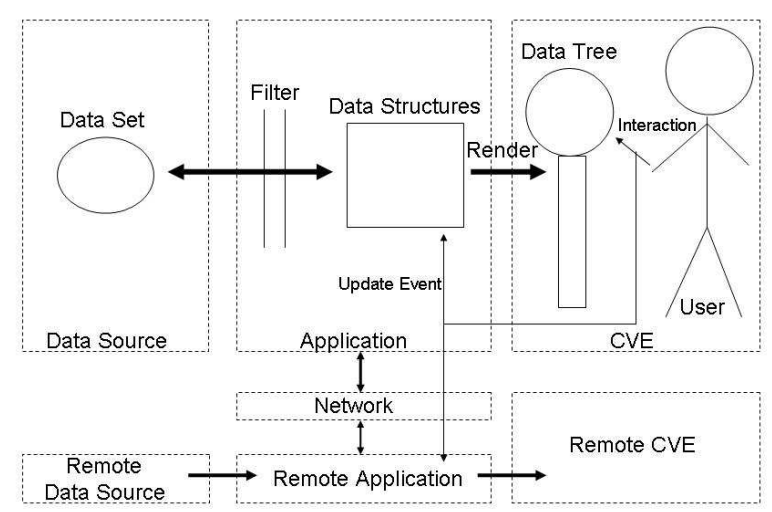

Fig. 2. Overview of Data Forest Structure

\section{Application Areas}

There are numerous areas that would benefit from using a Data Forest approach. The following areas were chosen due to their diversity of data, and in which further work is taking place.

\subsection{Stock Market Data}

The daily movement of the stock market, with its wide range of different parameters produces a vast amount of time dependant data. The ability to analyse this movement correctly and predict future changes has the potential to generate a considerable amount of money. Therefore this data is subject to many different traditional visualization techniques (e.g. 2D graphs, spreadsheets). Our approach is more suited to what would be considered off-line (i.e. non real-time) studies, but with some of the improvements mentioned in the future work it could then have the potential to move to real time use.

The test application was developed to visualize this type of data. This example was chosen to generate the forest, due to the different number of parameters that would be found in the data set. An example of some of the potential parameters that could be in the data set are share price, volume of trades, whether the share provides a dividend and how much, the percentage yield, the capital appreciation of the company, total number of shares in company, etc. Some of these parameters are highly dynamic (e.g. share price, volume of trades), but others are of a static nature for a long period of time (e.g. total number of share, amount of dividend). Therefore it is possible to match the movement of share prices with the changing shape/growth of the forest.

By utilising the structure proposed in CVV a collaborative version has been developed, remote users are represented by avatars and changes in one environment are then reflected in the other users environment by using event messages. A text grid is laid over the forest so that it is possible to associate each tree 
with a particular company (i.e. a tree with a NGC text label above it is referring to the National Grid Company). The forest is laid out alphabetically grid to ensure easier navigation if a user is interested in a particular company. The main methods of interaction with the Data Forest environment are through the use of 3D menus and an input device (e.g. a wand in an immersive environment). The use of 3D menus allows the user to easily interact with the data and environment. Currently the menus contain the following functionality:-

Stop/Start Simulation. Changing the forest from static mode to a dynamic or simulation mode (e.g. steps through the time dependant data). In simulation mode the forest is changed every set time period to reflect the new time variant data. It is possible to pause/stop the simulation at any time; also there is a step forward/back one time period function. This can be useful when the user discovers an interesting feature in the forest.

Increase/Decrease Size. Scaling the size of the forest to allow for different methods of analysis, scaling down gives the users a better overview of the complete forest while scaling up allows for more natural interaction and exploration of the data (i.e. the trees could be made life size).

Examine Tree. If the user decides to examine a particular data tree in more detail (i.e. exposing the roots of the tree), all that is required in that the user touches the desired tree with their input device (see Figure 3(a)) and then selects the examine function on the 3D menu. A new forest is rendered based on any related data to that tree. Once the user is finished with this requirement (see Figure $3(\mathrm{~b})$ ), they can return to the previous forest.

Predict Tree. While in the examine mode it is possible to carry out further analysis, with modules such as the predict tree function. This calculates an average crown and trunk value for this particular forest and then creates a tree based on these values. This is then added to the forest. Currently this is a very simple mathematical approach, but this could easily become a more complex algorithm.

\subsection{Network Data}

Computer networks are constantly being monitored and analysed to ensure the efficient transfer of data over the network. The data produced requires a method of visualization that will allow for time dependant analysis. By changing the axis of the Data Forest from vertical to horizontal we can use the forest to represent a computer network. The different parameters that could create a data tree are different network bandwidths, number of servers on the system, number of users connected to a server, load on server, amount of memory available and consumed at any one time by a server. This could be visualized in real time as discussed in the previous section or used for historical analysis. Another area of use can be as a forensic tool to simulate network conditions at the time of failure, so the user could navigate around the forest and examine all the factors that could 


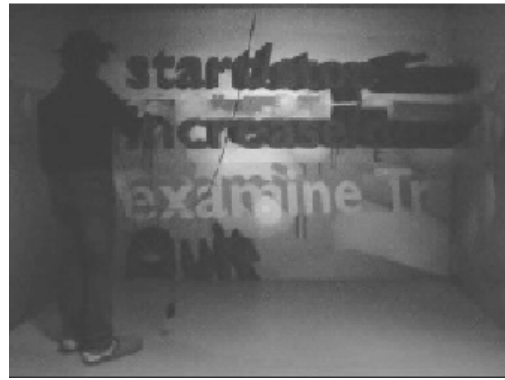

(a)

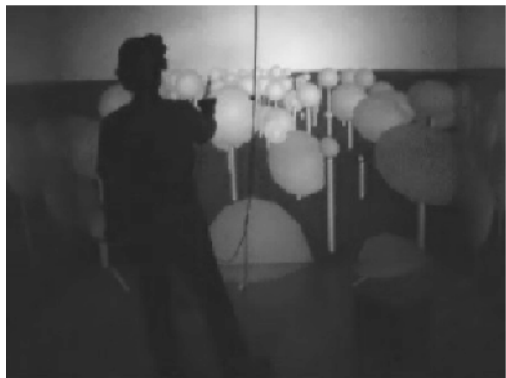

(b)

Fig. 3. (a) User selecting tree $3 \mathrm{D}$ menu (b) Underlying Data

have contributed to the problem. Companies are always interested in maximising their network usage; therefore they must visualize their current structure and flows. Similar functionality used in the stock market application could be reused here via the $3 \mathrm{D}$ menus.

\subsection{Marketing Data}

Consumers are continually producing data of interest through their shopping habits and preferences, to manufacturing companies who collect and store the data. Advances in methods of gathering this data, like the use of radio frequency ID (RFID) tags on products has increased the availability and complexity of this data. Therefore the data is extremely large and multi-variable. Analysing this is the focus of marketing departments within these companies. The data is extremely important to them, but it also has a very short life span. This is due to the short time to market of products and a fast changing customer demand. Therefore any insights into the shopping behaviour of customers is vital. An example of this could be point of sale data, it would produce parameters that would consist of frequency of purchase, cost of purchase, number of associated purchases, profit margin on purchase, purchased from and many more. This data is not as time dependant as the other examples but analysing the purchasing decision of customers using traditional methods is complex and not intuitive. Therefore this is another area that could use a Data Forest, with similar features developed in the test application.

\section{Conclusion and Future Work}

This paper has successfully outlined the concept of a Data Forest, which is a new and novel approach that uses VR to visualising data that does not easily fit traditional methods. A test application has been developed utilising stock market data and a collaborative version has been successfully incorporated into the collaborative structure proposed by CVV. Our modular approach is flexible 
and allows for the reuse of different function within different contexts, by just changing modules, different types of data sets could be loaded into our environment.

Future work will include incorporating Really Simple Syndication (RSS) feeds directly in the collaborative environment in the form of a scrolling text bar. The option to extract this data from the RSS feed and utilise it in updating the relevant data trees accordingly will be investigated. This will allow for real-time information to be incorporated into our Data Forest. The inclusion of virtual maps of the forest for large environments so the users can see the position of the remote user (i.e. out of visual range of the user), therefore allowing a larger user search area will be researched. We aim to investigate allowing the user to specify how they would like the forest to be arranged to enable greater ease in searching the forest for particular areas of interest (e.g. in the stock market application the user could group the trees according to industries).

\section{References}

1. C.Carlsson et al, "DIVE: a platform for multi-user virtual environment", Computer E Graphics, 1993, p663-669, Vol .17, No.6.

2. Coanim, "Electronic Visualisation Laboratory (EVL), University of Illinois at Chicago", 2005, http://www.evl.uic.edu/cavern/agave/coanim.html.

3. C.Cruz-Neria et al, "The CAVE: audio visual experience automatic environment", Communication of the ACM, 1992, p64-72, Vol.35, No.6.

4. D. Foulser, "Iris Explorer - a framework for investigation", Computer Graphics, 1995, 29(2):13-16

5. R. Jamieson et al, "Collaborative Virtual Visulaization", First Austian Grid Symposium, Austira, 2005

6. J.Leigh et al, "CAVERN: A Distributed Architecture for Supporting Scalable Persistence and Interoperability in Collaborative Virtual Environments", Journal of Virtual Reality Research, Development and Applications, 1997, p261-296, Vol.2.2.

7. B. Lucas et al, "An architecture for a scientific visualization system", Proceddings of Visualization '92, 1992, p107-114

8. V.Normand et al, "Collaborative Virtual Environments: the COVEN Project.", Proc.of the Framework for Immersive Virtual Environments Conf. (FIVE), 1996.

9. W.J. Schroeder et al. "The design and implementation of an object-oriented toolkit for 3d graphics and visualization." Proc. of Visualization,ACM, 1996, p93-100.

10. SGI Inc, "MineSet: a system for high-end data mining and visualization", Int. Conf. On Very Large Databases (VLDB'96), Bombay, India, 1996, p595

11. S.Singhal et al, "Networked Virtual Environments: Design and Implementation", ACM Press, 1999.

12. C. Upson et al. "The Application Visualization System: a computational environment for scientific visualization", Computer Graphics and Applications, 1989, $9(4): 30-42$ 